\title{
County-level Analysis of Perinatal Health Indicators Within a Single Health System Catchment
}

\section{Dominick J. Lemas ( $\nabla$ djlemas@ufl.edu )}

University of Florida

\section{Claire Layton}

University of Florida

\section{Hailey Ballard}

University of Florida

$\mathrm{KeXu}$

University of Florida

John C. Smulian

University of Florida

\section{Matthew Gurka}

University of Florida

\section{Matthew Shane Loop}

University of North Carolina at Chapel Hill

\section{Erica L. Smith}

University of Florida

\section{Callie F. Reeder}

University of Florida

\section{Adetola Louis-Jacques}

University of Florida

\section{Chu J Hsiao}

University of Florida

\section{Nicole Cacho}

University of Florida

\section{Jaclyn Hall}

University of Florida

\section{Research Article}

Keywords: Adverse perinatal health outcomes, Social determinants of health (SDoH), County Health Rankings, The University of Florida Health Perinatal Catchment Area (UFHPCA), breastfeeding.

Posted Date: December 28th, 2021

DOI: https://doi.org/10.21203/rs.3.rs-1183907/v1

License: (c) This work is licensed under a Creative Commons Attribution 4.0 International License. Read Full License 


\section{Abstract}

Background: Adverse perinatal health outcomes are disproportionally impacted in rural communities. Social determinants of health (SDoH) defined by nonclinical social, behavioral, and economic factors may impact up to $90 \%$ of health outcomes in rural communities.

Objective: To evaluate county-level perinatal patterns in health outcomes, health behaviors, socioeconomic vulnerability, and healthcare providers across rural and non-rural Florida counties within a single health system catchment.

Methods: Socioeconomic vulnerability metrics, digital connectivity, licensed provider metrics, and behavioral data and were obtained from Floridahealthcharts.com and the County Health Rankings. County-level birth and perinatal health outcome data were obtained from the Florida Department of Health. The University of Florida Health Perinatal Catchment Area (UFHPCA) was defined as all Florida counties where $\geq 5 \%$ of all infants were delivered at Shands Hospital in Alachua county between June 2011 and April 2017. County-level rurality was determined by Florida Statutes 288.0656 rurality designations.

Results: The UFHPCA included three non-rural and ten rural counties that represented more than 64,000 deliveries over a 5year 9 -month period. We found that nearly 1 in 3 infants resided in a rural county $(n=20,899)$, and 7 out of 13 counties did not have a licensed obstetrician gynecologist. Nine counties reported maternal death rates that were between 1 and 4 -fold higher than the statewide rate, and rural counties generally reported neonatal mortality and preterm birth rates that were higher than the statewide averages. We found maternal smoking rates (range $6.8 \%-24.8 \%$ ) were above the statewide rate $(6.2 \%)$ for all counties in the catchment. Except for Alachua county, breastfeeding initiation rates (range $54.9 \%$ $81.4 \%$ ) and access to household computing devices (range $72.8 \%-86.4 \%)$ were below the statewide rate $(82.9 \%$ and $87.9 \%$, respectively). Finally, we found that childhood poverty rates (range $16.3 \%-36.9 \%$ ) in our catchment was above the statewide rate (18.5\%), except for Suwanee and Columbia counties.

Conclusions: The health burden of the UFHPCA is characterized by both rural and non-rural counties with increased maternal and neonatal death and preterm birth, as well as adverse health behaviors that include smoking during pregnancy and lower levels of breastfeeding.

\section{Introduction}

Adverse perinatal health outcomes including maternal and infant mortality are far higher in the United States than in similarly large and wealthy countries $(1,2)$. In 2017, the World Health Organization reported that the US was one of only two countries to report a significant increase in maternal mortality since 2000 (3). Despite significant advances in clinical care (7-9), there is a growing awareness that up to $90 \%$ of health outcomes are the result of nonclinical factors -known as the social determinants of health (SDoH)- which include social, behavioral and economic domains (10). Perinatal health disparities are more likely among women who are young, low-income, African American, unmarried, less educated, receiving federal assistance (i.e., The Special Supplemental Nutrition Program for Women, Infants, and Children (WIC)), are overweight or obese before pregnancy, or reported their pregnancy was unintended (4). Maternal healthcare spans pregnancy through the postpartum period (5) and prenatal care is essential for a healthy pregnancy and delivery (6). Among mothers who did not receive prenatal care, low birth weight of their infant is three to five times more likely to occur, and infant death following delivery is five times more likely to occur compared to those mothers who receive adequate prenatal care (2).

Common social determinants that are used to explain disparities in health outcomes are income, unemployment, education, racial or ethnicity, or geography (11). In many areas of the US, the racial discrimination experienced by minority populations is negatively associated with health outcomes, and the prevalence of adverse health outcomes throughout childhood often persists despite increasing income and education levels $(12,13)$. The impact of SDoH on health outcomes 
is likely mediated through a complex interaction among health behaviors, clinical care, social and economic factors, and an individual's physical environment (10). As an example, maternal education and household income (14) are important modifiers of perinatal health behaviors such as maternal smoking $(15,16)$ and breastfeeding success $(17)$. Maternal education across all racial groups remains the strongest factor that influences breastfeeding initiation and duration (18) and smoking cessation success (16). Nearly twice as many higher-income women breastfeed exclusively at six months as compared to their lower-income counterparts (19). With respect to maternal smoking, low socioeconomic status was associated with higher risk of smoking at the time of conception and continued smoking during pregnancy (15). Taken together, SDoH at the individual-level include factors such as education and income that occur outside of the health care provider's office and impact perinatal health outcomes.

We defined and examined a perinatal health catchment area for University of Florida (UF) Health Shands Hospital, a single health care system with broad regional reach in north central Florida, in order to identify opportunities to improve health related to social determinants. Defining a geographical area around a healthcare service point is important to not only estimate community needs but also facilitate planning of healthcare initiatives (20). We characterized county-level patterns in perinatal health outcomes and behaviors, socioeconomic vulnerability, and the availability of healthcare providers across rural and non-rural Florida counties within this single health system catchment. The long-term goal of the UFHPCA is to facilitate collaborations in which researchers, providers, public health practitioners, and nonprofit organizations can utilize the data to develop or expand applied perinatal research, planning, and implementation, with an emphasis on improving local health outcomes.

\section{Data And Methods}

Perinatal Catchment Definition: The UFHPCA was defined at the county level from births occurring at UF Health Shands Hospital. We obtained electronic health records (EHR) from UF Health Shands Hospital that included all live births occurring between June 2011 and April 2017 (21). Linkage of maternal-infant records to obtain mother's residential 5 or 9 digit zip code was completed by an honest broker within the University of Florida Health Integrated Data Repository (IDR) before releasing the de-identified data to the research team. ZIP codes from the delivery encounters were available on $99.1 \%$ of records. Nine-digit and most 5-digit zip codes were linked to county. The number of births from 5-digit zip codes that cross county borders were aggregated to the county level using the 2010 ZIP Code Tabulation Area (ZCTA) to County Relationship File from the U.S. Census Bureau (22). UF Health EHR birth counts per county were compared to county birth counts from the Florida Bureau of Vital Statistics during the same period (June 2011 to April 2017). The defined the UFHPCA as all counties where $5 \%$ or more of county births occurred at UF Health. Supplementary Figure 1 outlines selection of EHR records included in this study. The EHR record data was used purely to define the UFHPCA boundary.

State and Local Data: Socioeconomic vulnerability metrics, digital connectivity, licensed provider metrics, and behavioral data and were obtained from Floridahealthcharts.com and the County Health Rankings (CHR). County level birth and perinatal health outcome data were obtained from the Florida Department of Health.(23) To match the time of the EHR records, rates for each measure were calculated from FL Health Charts' annual rates between 2011-2017 and weighted by county-level annual population. Counts for each year's numerator and denominator were obtained and aggregated to generate one rate for June 2011 to April 2017. All data visualization and tables were created using R statistical software (24). FL Health Charts provided rates of computing device access beginning in 2017. The $2021 \mathrm{CHR}$ z-scores were obtained for all 67 Florida counties as an established framework to rank each county within Florida from most to least healthy (25).

Primary Outcomes: Perinatal health outcomes included maternal mortality, neonatal mortality, and preterm birth. Behavioral outcomes included breastfeeding initiation rates and non-smoking rates during pregnancy. Socioeconomic vulnerability metrics included rates of families with related children younger than 5 years above the poverty line and rates 
of households with one or more computing devices. Healthcare access included number of licensed pediatricians, obstetrician gynecologists (OB-GYNs), and family practice physicians.

Rurality Designations: Rurality on the county level was identified according to rurality designations defined by Florida Statutes 288.0656. A county was designated as rural if the population did not exceed 75,000, or if the population did not exceed 125,000 and was contiguous to a county with a population of less than 75,000 (26).

Analysis: Once counties meeting the threshold to be designated within the UFHPCA were identified, county-level rates of perinatal behaviors, socioeconomic vulnerability metrics, and licensed provider metrics were compared to state-wide rates. The ggplot2 and grid packages were used to visualize rates geographically by county $(27,28)$. County rates were ranked by rates for interpretation.

\section{Results}

UF Health Shands Hospital delivered 16,711 infants between June 2011 and April 2017. Of those infants, 112 were unable to be linked to a mother and excluded from the study. Zip code data was missing for a further 43 infants, and the

remaining 16,566 infants were linked to a county of residence (Supplementary Figure 1). Out of 67 counties in the state of Florida, we identified the perinatal catchment area as 13 counties where $\geq 5 \%$ of infants within the county were delivered within the UF Health system (Table 1). Counties included in the UFHPCA were Alachua, Citrus, Marion, Putnam, Bradford, Columbia, Union, Suwannee, Gilchrist, Levy, Hamilton, Dixie, and Lafayette. The UFHPCA included three non-rural counties and ten rural counties.

\section{Table 1. County-Level Demographics of UF Health Perinatal Catchment Area}


Geographic Features

Population Demographics

Birth Data

(\% of Population in 2017)

(June 2011 -

April 2017)

\begin{tabular}{|c|c|c|c|c|c|c|c|c|c|c|}
\hline County & Rurality & Density & Population & $\begin{array}{l}\mathrm{NH} \\
\text { White }\end{array}$ & $\begin{array}{l}\mathrm{NH} \\
\text { Black }\end{array}$ & $\begin{array}{l}\mathrm{NH} \\
\text { Other }\end{array}$ & Hispanic & $\begin{array}{l}\text { Females } \\
18-35\end{array}$ & $\begin{array}{l}\text { Live } \\
\text { Births }\end{array}$ & $\begin{array}{l}\text { Shands } \\
\text { Births } \\
(\%)\end{array}$ \\
\hline Alachua & $\begin{array}{l}\text { Non- } \\
\text { Rural }\end{array}$ & 297.0 & 259349 & 62.0 & 20.0 & 8.6 & 9.4 & 19.4 & 17034 & 42.3 \\
\hline Citrus & $\begin{array}{l}\text { Non- } \\
\text { Rural }\end{array}$ & 243.0 & 144922 & 88.5 & 2.8 & 3.3 & 5.4 & 6.9 & 6161 & 5.5 \\
\hline Marion & $\begin{array}{l}\text { Non- } \\
\text { Rural }\end{array}$ & 217.0 & 352067 & 71.4 & 12.5 & 3.6 & 12.5 & 9.3 & 20200 & 18.5 \\
\hline Putnam & Rural & 99.6 & 73068 & 71.8 & 15.9 & 2.6 & 9.8 & 9.5 & 4933 & 10.0 \\
\hline Bradford & Rural & 91.1 & 27808 & 74.3 & 19.0 & 2.8 & 3.9 & 9.2 & 1789 & 26.5 \\
\hline Columbia & Rural & 85.9 & 69250 & 72.9 & 17.8 & 3.3 & 6.0 & 10.0 & 4777 & 13.4 \\
\hline Union & Rural & 62.8 & 15896 & 69.4 & 22.4 & 2.5 & 5.7 & 7.4 & 961 & 23.1 \\
\hline Suwannee & Rural & 63.6 & 44527 & 75.7 & 12.9 & 2.3 & 9.1 & 9.0 & 2718 & 16.6 \\
\hline Gilchrist & Rural & 49.2 & 16977 & 87.4 & 4.6 & 2.4 & 5.6 & 9.2 & 1131 & 36.5 \\
\hline Levy & Rural & 35.5 & 40832 & 80.1 & 9.0 & 2.7 & 8.3 & 9.4 & 2324 & 45.9 \\
\hline Hamilton & Rural & 27.7 & 14749 & 55.0 & 33.2 & 2.8 & 9.1 & 7.9 & 911 & 16.8 \\
\hline Dixie & Rural & 23,2 & 17040 & 84.0 & 9.6 & 2.3 & 4.2 & 7.7 & 925 & 42.5 \\
\hline Lafayette & Rural & 16.0 & 8651 & 71.1 & 14.5 & 2.1 & 12.4 & 8.0 & 430 & 29.9 \\
\hline
\end{tabular}

Note: Population Density retrieved from U.S. Census Bureau 2010 10-year Estimate. Population Density measured in square miles. Population and demographic data provided by FLHealthCharts.com, which is managed by the Florida Department of Health, Division of Public Health Statistics \& Performance Management.

$\mathrm{NH}=$ Non-Hispanic .

Analysis from the County Health Rankings revealed that twelve out of thirteen counties in the UFHPCA had a positive health outcomes z-score, indicating a high health burden compared to the state rate (Figure 1). According to the Florida Department of Health, the total population of the UFHPCA in 2017 was 1,085,136. During the study period, there were 64,294 live births with $32.5 \%(20,899)$ of deliveries coming from rural counties. The percentage of live births at UF Health Shands Hospital across the UFHPCA ranged from 5.5\% in Citrus County to $45.9 \%$ in Levy County (Table 1). Alachua, Bradford, Gilchrest, Levy, Dixie, and Lafayette all had approximately 30\% or more of live births at Shands Hospital.

We found that four counties in our analysis reported no maternal deaths during the period studies; however, the remaining nine counties reported age-adjusted maternal death rates that were 1 to 4 -fold higher than the statewide rate of $2.1 \%$ deaths per 100,000 population (Table 2). With respect to infant mortality, we found that twelve of the thirteen counties in the UFHPCA reported mortality rates (6.4 - 13.1 deaths per 1,000 live births) that were above the state-wide rate (6.1 deaths per 1,000 live births). We also found that preterm birth rates (Figure 2, 9.0 - 13.1 preterm infants per 1,000 live births) among the UFHPCA counties were above the state-wide rate of 10.1 infants per 1,000 live births except for Marion, Citrus, and Gilchrist counties which had rates of 9.0,10.1, and 9.0 respectively. All counties in the UFHPCA had maternal smoking rates (Figure 3, 6.8\% - 24.8\%) above the statewide rate (6.2\%). Within the UFHPCA, only Alachua County $(84.3 \%)$ had a 
higher breastfeeding initiation rate than the statewide rate (82.9\%), with the remaining catchment counties' rates ranging from $52.8 \%$ to $81.4 \%$ (Figure 4).

\section{Table 2. UF Health Perinatal Catchment Area Health Outcomes and Health Behaviors}

\begin{tabular}{|c|c|c|c|c|c|}
\hline & \multicolumn{3}{|c|}{ Perinatal Health Outcomes } & \multicolumn{2}{|l|}{ Perinatal Behaviors } \\
\hline Area & $\begin{array}{l}\text { Maternal Deaths (Age- } \\
\text { adjusted rate per } \\
100,000 \text { population) }\end{array}$ & $\begin{array}{l}\text { Infant } \\
\text { Mortality (Per } \\
1,000 \text { live } \\
\text { births) }\end{array}$ & $\begin{array}{l}\text { Preterm } \\
\text { Births (Per } \\
1,000 \text { live } \\
\text { births) }\end{array}$ & $\begin{array}{l}\text { Births to Smoking } \\
\text { Mothers During } \\
\text { Pregnancy }(\%)\end{array}$ & $\begin{array}{l}\text { Births to Mothers } \\
\text { Who Initiate } \\
\text { Breastfeeding (\%) }\end{array}$ \\
\hline Florida & 2.1 & 6.1 & 10.1 & 6.2 & 82.9 \\
\hline Alachua & 3.0 & 8.0 & 10.4 & 6.8 & 84.6 \\
\hline Citrus & 2.4 & 7.1 & 10.1 & 23.0 & 74.2 \\
\hline Marion & 2.2 & 8.1 & 9.0 & 13.5 & 74.9 \\
\hline Putnam & 5.5 & 8.0 & 11.8 & 20.2 & 75.3 \\
\hline Bradford & 4.8 & 11.9 & 11.6 & 21.9 & 71.4 \\
\hline Columbia & 2.1 & 9.1 & 11.0 & 17.6 & 68.4 \\
\hline Union & 0.0 & 4.1 & 13.1 & 20.9 & 70.1 \\
\hline Suwannee & 0.0 & 7.9 & 11.3 & 16.3 & 70.4 \\
\hline Gilchrist & 0.0 & 7.0 & 9.0 & 15.5 & 81.4 \\
\hline Levy & 3.3 & 7.3 & 11.1 & 18.2 & 78.6 \\
\hline Hamilton & 8.8 & 10.6 & 10.5 & 15.2 & 54.9 \\
\hline Dixie & 9.1 & 6.4 & 11.9 & 24.8 & 70.1 \\
\hline Lafayette & 0.0 & 6.0 & 11.9 & 16.3 & 73.2 \\
\hline
\end{tabular}

Note: Data provided by FLHealthCharts.com, which is managed by the Florida Department of Health, Division of Public Health Statistics \& Performance Management. Data captures only actively licensed providers for each fiscal year July 1 June 30 during the 2011 to 2017 period.

All counties other than Columbia (17.6\%) and Suwanee (16.3\%) in the catchment had childhood poverty rates that exceeded the statewide rate of $18.5 \%$ of families with young children living in poverty (Table 3, Figure 5, 20.7\% - 36.9\%). All counties other than Alachua county (89.0\%) in the UFHPCA had lower rates of access to computing devices in 2017 than the statewide rate (87.9\%), (Figure 6, 60.5\% - 86.4\%). Three rural counties with the lowest access to computing devices in 2017 were Union (72.7\%), Bradford (72.8\%), and Dixie (69.5\%) counties. Seven rural counties in the UFHPCA did not have a licensed OB-GYN in the entire county. Twelve out of thirteen counties in the UFHPCA were below the statewide rate for at 
least one type of provider during the period studied. For the thirteen counties in the UFHPCA, ten had fewer than $50 \%$ of the state average density for licensed pediatricians, seven had fewer than $50 \%$ of the state average density for family practice physicians, and nine had fewer than $50 \%$ of the state average density for OB-GYNs.

\section{Table 3. UF Health Perinatal Catchment Area Socioeconomic Vulnerability and Healthcare Access}

Socioeconomic Vulnerability

(\%) years) Below the Poverty Line
Area $\quad$ Families with Related Children $(<5$
Households with One or More Computing Devices
Licensed Providers Available

(Per 100,000 population)

$\begin{array}{lll}\text { Pediatricians } & \text { Family } & \text { OB- } \\ & \text { Practice } & \text { GYNs } \\ & \text { Physicians } & \end{array}$

\begin{tabular}{llll}
87.9 & 19.6 & 21.4 & 9.7 \\
89.0 & 64.6 & 45.8 & 21.4 \\
86.4 & 7.0 & 21.5 & 4.0 \\
83.5 & 8.2 & 18.1 & 4.8 \\
75.9 & 8.5 & 11.2 & 9.1 \\
\hline 72.8 & 1.8 & 15.2 & 0.6 \\
85.5 & 5.9 & 15.7 & 2.0
\end{tabular}

Columbia

17.5

87

Alachua

20.7

26.7

26.1

85.5

72.7

1.1

8.5

3.8

0.0

82.4

0.4

78.2

6.9

5.9

0.0

79.7

1.7

13.2

0.0

76.7

0.0

2.3

0.0

Hamilton

33.7

Dixie

24.8

69.5

4.1

7.1

0.0

Lafayette

77.7

0.0

1.9

0.0

Note: Data provided by FLHealthCharts.com, which is managed by the Florida Department of Health, Division of Public Health Statistics \& Performance Management. Data captures only actively licensed providers for each fiscal year July 1 June 30 during the 2011 to 2017 period.

\section{Discussion}

Summary: Geographic variation in health outcomes has consistently demonstrated that rural communities are disproportionately affected (29-32) in the domains of mortality and clinical care (33). This study identified thirteen Florida counties as part of the UFHPCA which included rural and non-rural counties, representing more than 64,000 deliveries over nearly five years. Twelve out of thirteen counties in the UFHPCA had a higher health burden compared to the state rate, while three catchment counties were in the top five Florida counties for overall health burden. Our analysis 
revealed that nearly 1 in 3 infants (32.5\%) within the UFHPCA reside in a rural county. We also found counties that reported a 4-fold increase in maternal death rates relative to the statewide average. Rural counties in our analysis reported neonatal mortality and preterm birth rates that were generally higher than the statewide averages. Rural counties within the catchment were characterized by higher levels of smoking during pregnancy, increased childhood poverty rates, lower levels of breastfeeding initiation, and more limited access to computing devices when compared to the state of Florida as a whole. Analysis of perinatal health care providers revealed that rural counties had fewer licensed providers in the areas of family medicine, obstetrics, and pediatricians. Collectively, our results revealed that relative to statewide averages, North Central Florida is characterized by both rural and non-rural counties with higher maternal and infant mortality as well as higher levels of smoking during pregnancy and childhood poverty and lower levels of breastfeeding.

Health Care Access: A perinatal healthcare desert is defined as geographic areas that lack adequate obstetric health services (34). In 2014, less than half of all rural counties in the US had hospital-based obstetric care (35), emphasizing the impact of socioeconomic status and transport resources (36) on adequate health care services for rural populations. An important observation made by this study is that seven out of thirteen counties in our analysis did not have a licensed obstetrician gynecologist, suggesting that patients living in these areas may reside within a perinatal healthcare desert. Women living in perinatal healthcare deserts experience much higher rates of adverse outcomes during pregnancy and childbirth compared to those living outside these areas (37). In Louisiana from 2016-2017, women living in perinatal healthcare deserts experienced a $91 \%$ increase in risk of maternal mortality during pregnancy and up to one year postpartum, regardless of the women's age, race or ethnicity, or socioeconomic status. Our results were generally consistent with these observations, whereby maternal mortality was the highest in counties without a licensed obstetrician gynecologist. Given the complexity of healthcare accessibility in rural communities $(38,39)$, our results highlight the importance of defining "high-risk" areas that can inform precision public health interventions focused on reducing maternal mortality in rural communities (40).

Maternal Mortality: The US consistently ranks last in maternal mortality among industrialized nations, with approximately 700 women each year dying due to pregnancy or delivery complications (2). Kozhimannil et al found that rural residents had a 9 percent greater probability of severe maternal morbidity and mortality during pregnancy, compared with urban residents (41). Moreover, estimates suggest that nearly 3 in 5 pregnancy-related deaths in the US are potentially preventable and this proportion can vary, with some cases indicating that up to $90 \%$ of maternal deaths may be preventable (42-44). We found that four counties in our analysis reported no maternal deaths during the period studied; however, the remaining nine counties reported age-adjusted maternal death rates that were 1 to 4 -fold higher than the statewide rate of $2.1 \%$ deaths per 100,000 population. Previous work in Florida has demonstrated that overall public health expenditures were not directly associated with improvements perinatal health outcomes such as maternal deaths (45). Specifically, Bernet et al. reported that pregnancy-related public health programs across all 67 Florida counties resulted in statistically significant reductions in high-risk groups that included the black maternal death rate and the black-white maternal death gap after adjusting for income, employment and access to care (45). Although these analyses did not consider rurality, clinical efforts to reduce maternal deaths in rural communities includes the development of evidence-based clinical practices called maternal safety bundles that are designed to reduce the incidence of and risks associated with severe maternal morbidity and mortality (41). Taken together, public health policies and clinical practices that aim to address maternal mortality should ensure the representation of rural residents within decision-making bodies and public health interventions (46).

Health Behaviors: Maternal health behaviors during pregnancy and delivery are key determinants of infant outcomes. Our study revealed that all counties in the UFHPCA had maternal smoking rates that were higher than the statewide average. Prenatal smoking increases risks of adverse pregnancy outcomes, including stillbirth (47), preterm birth (48), and infant mortality (49). We also found that 12 counties in the UFHPCA had lower breastfeeding initiation rates than the statewide average, with the exception of Alachua county. As advised in the Center for Disease Control's Breastfeeding Report Card, 
2020, individualized support in the first few hours and days is critical to help mothers meet their breastfeeding goals. Our analysis also revealed that rural counties generally reported neonatal mortality and preterm birth rates that were higher than the statewide averages. Family poverty, another $\mathrm{SDoH}$, influences many aspects of perinatal health, including maternal diet and exercise and access to perinatal care. Poverty is one component of socioeconomic status, which has also been intimately associated with birth weight (50). Birth weight is tied to many outcomes during the perinatal period and long-term health (51). Higher poverty is associated with a lower birth weight (52). Our study revealed that every county in the UFHPCA exceeded the Florida statewide rate of poverty. Taken together, our results demonstrate that UFHPCA counties had some of the lowest breastfeeding initiation rates across the state of Florida which highlights the need for innovative public health interventions that promote breastfeeding in rural and non-rural areas. For example, a metaanalysis identified smoking as an important factor for breastfeeding, where nonsmoking mothers had higher breast feeding initiation and continuation rates (53). Recognizing that many of the counties in the UFHPCA had both higher maternal smoking rates and lower breastfeeding initiation rates, an intervention that aims to lower smoking rates could improve both smoking and breastfeeding behaviors.

Telemedicine: With the increasing utilization of telemedicine throughout the United States, many states have begun integrating telemedicine into obstetrics and maternal health clinics. Access to telemedicine services could be an effective technique to improve maternal health outcomes for at-risk populations in rural areas such as many counties within the UFHPCA. Long driving times to reach physicians and limited options for perinatal care and digital information in rural counties may be detrimental to maternal health outcomes (37). In 2011, The Georgia Department of Public Health partnered with health districts and maternal medicine specialists to provide telemedicine consultations for African American and Hispanic women with high-risk pregnancies. In an 18-month period the preterm labor rate was reduced in the target population from $18.8 \%$ to $8 \%$ across 500 pregnancies. Additionally, there was a noticeable increase in the adherence to follow-up care following delivery (54). Telemedicine services allowed patients to communicate with their physicians via computers, phones, or tablets (55). Health interventions that leverage telemedicine have been associated with improvements in obstetrics outcomes (56). The limitations of UFHPCA regarding provider availability in maternity deserts highlight the need for innovative maternity care. Telemedicine could potentially bridge this gap in care but the limitation of computer access within the UFHPCA will prove another unique challenge.

Strengths and Limitations: The UFHPCA defines a region of North Central Florida. The strengths of using county rankings as a primary metric for comparison include ease of interpretation and visualization of unmet medical needs that can help generate active stakeholder participation in appropriate public health and policy actions. Because the data is systematically collected through existing state and federal agencies, the broader impact of interventions can be readily assessed with new data pulls. However, using existing county-level data limits the resolution of data that can be achieved. For example, while the available data allows us to count the number of licensed obstetrician gynecologists by county, it prevents us from capturing obstetric care offered by other providers (e.g. family medicine physicians who provide obstetric care, nurse practitioners, or midwives). Likewise, although we were able to compare rural and non-rural counties, such a binary designation may not capture local neighborhood effects. Given that the socioeconomic heterogeneity of rural areas has been documented (57), future studies on the UFHPCA should look beyond the binary rurality designation of each county and examine health outcomes related to poverty and other neighborhood demographic characteristics using census tract level investigations. Additionally, the only perinatal health behaviors measured were maternal smoking and breastfeeding initiation. Future studies could include a more comprehensive list of behaviors to provide a more descriptive picture. A higher resolution understanding of perinatal health outcomes and geographical locations of pediatricians, OBGYNs, family practice physicians, and other perinatal care providers within the UFHPCA may help enhance understanding of the areas with the highest perinatal health burden and identify areas where public health initiatives would provide the most benefit. Alachua, location of UF Health Shands Hospital at the University of Florida, is the only county in the UFHPCA that is above the Florida rate for average numbers of pediatricians, OB-GYNs, and family practice physicians per 
100,000 population. This further illustrates that UF Health Shands Hospital, the regional referral center for North Florida, serves an vulnerable population that often does not currently receive adequate care in their county of residence.

\section{Conclusion}

The results of this study highlight the importance of understanding the effects of SDoH on healthcare accessibility and perinatal outcomes in rural and non-rural counties within an accurate service catchment area. Delineating the boundaries of the UFHPCA allows researchers and providers to focus primary data collection and intervention implementation within an actionable region, facilitating collaborations with the appropriate non-profits and policy-makers to work towards the goal of addressing health disparities and communication inequalities. This paper therefore represents an essential first step towards launching interventions to address the impact of SDoH on perinatal health. These findings provide additional insight into prioritizing strategies that address SDoH and expand healthcare access to rural counties, such as improving healthcare access via telemedicine. By understanding how health behaviors and socioeconomic factors juxtapose with the physical environments in which patients live (i.e. rurality), the findings help identify potential policies and programs that may improve perinatal outcomes.

\section{Abbreviations}

- CHR: The County Health Rankings

- EHR: Electronic Health Records

- ER: Emergency Room

- $\mathrm{NH}$ : Non-Hispanic

- OB-GYN: Obstetricians and Gynecologists

- SDoH: Social Determinants of Health

- UF: University of Florida

- UFHPCA: The University of Florida Health Perinatal Catchment Area

- WIC: The Special Supplemental Nutrition Program for Women, Infants, and Children

- ZCTA: ZIP Code Tabulation Area

\section{Declarations}

\section{Ethics approval and consent to participate}

The EHR data for this study was approved by the Institutional Review Board of the University of Florida (IRB201601899).

\section{Consent for publication}

Not applicable.

\section{Availability of data and materials}

EHR data used in this study cannot be shared publicly in order to protect participant confidentiality. All requests to review data that may include potential subject and/or patient identifiers will be reviewed by our University's Privacy Office and Institutional Review Board to ensure adequate protection of subjects. Any dissemination approved by the University will be reviewed by a University honest data broker to ensure appropriate legal and ethical compliance with regulatory agencies. Requests for data should be addressed to Chris Harle at the University of Florida (charle@ufl.edu). County-level data on live birth rates, perinatal health data, and socioeconomic vulnerability metrics are publicly available from the Florida 
Department of Health's FL Health Charts, [http://www.flhealthcharts.com/Charts/MaternalAndChildHealth/default.aspx]. The CHR's Health Outcomes z-scores are also publicly available, [https://www.countyhealthrankings.org/explore-healthrankings/use-data]. The $\mathrm{R}$ code used to create all maps and tables displayed in this study is available in the GitHub repo "mombaby-ehr-SdoH" [https://github.com/lemaslab/mombaby-ehr-SdoH].

\section{Competing interests}

Not applicable

\section{Funding}

This work was supported by the National Institute of Diabetes and Digestive and Kidney Diseases [K01DK115632] and the University of Florida Clinical and Translational Science Institute [UL1TR001427]. The content is solely the responsibility of the authors and does not necessarily represent the official views the University of Florida's Clinical and Translational Science Institute or the National Institutes of Health.

\section{Authors' contributions}

All authors designed research (project conception, development of overall research plan, and study oversight); CL and HB analyzed data and performed statistical analysis; DJL, JH, ML, and KX interpreted data findings; $D J L, C L, H B$, and KX drafted the initial manuscript; all authors provided critical revision of the manuscript; DJL and JH had primary responsibility for final content. The funders had no role in study design, data collection and analysis, decision to publish, or preparation of the manuscript.

\section{References}

1. International Comparison | 2019 Annual Report [Internet]. America's Health Rankings. [cited 2021 Aug 19]. Available from: https://www.americashealthrankings.org/learn/reports/2019-annual-report/international-comparison

2. Racial/Ethnic Disparities in Pregnancy-Related Deaths - United States, 2007-2016 | MMWR [Internet]. [cited 2021 Aug 19]. Available from: https://www.cdc.gov/mmwr/volumes/68/wr/mm6835a3.htm

3. World Health Organization. Trends in maternal mortality 2000 to 2017: estimates by WHO, UNICEF, UNFPA, World Bank Group and the United Nations Population Division [Internet]. World Health Organization; 2019 [cited 2021 Aug 23]. 104 p. Available from: https://apps.who.int/iris/handle/10665/327595

4. Ahluwalia IB, Morrow B, Hsia J. Why do women stop breastfeeding? Findings from the Pregnancy Risk Assessment and Monitoring System. Pediatrics. 2005 Dec;116(6):1408-12.

5. Health Coverage Options for Pregnant or Soon to Be Pregnant Women | HealthCare.gov [Internet]. [cited 2021 Sep 29]. Available from: https://www.healthcare.gov/what-if-im-pregnant-or-plan-to-get-pregnant/

6. Prenatal care I Office on Women's Health [Internet]. [cited 2021 May 23]. Available from: https://www.womenshealth.gov/a-z-topics/prenatal-care

7. Kodama Y. The Preterm Newborn: Morbidity and Mortality-Including a Population-Based Study in Miyazaki Prefecture. In: Sameshima H, editor. Preterm Labor and Delivery [Internet]. Singapore: Springer; 2020 [cited 2021 Aug 24]. p. 215-22. (Comprehensive Gynecology and Obstetrics). Available from: https://doi.org/10.1007/978-981-139875-9_22

8. Duhig K, Vandermolen B, Shennan A. Recent advances in the diagnosis and management of pre-eclampsia. F1000Res. 2018 Feb 28;7:242.

9. Cheong JLY, Spittle AJ, Burnett AC, Anderson PJ, Doyle LW. Have outcomes following extremely preterm birth improved over time? Seminars in Fetal and Neonatal Medicine. 2020 Jun 1;25(3):101114. 
10. Hood CM, Gennuso KP, Swain GR, Catlin BB. County Health Rankings: Relationships Between Determinant Factors and Health Outcomes. Am J Prev Med. 2016 Feb;50(2):129-35.

11. Marmot M. Social determinants of health inequalities. Lancet. 2005 Mar 19;365(9464):1099-104.

12. Flanagan BE, Hallisey EJ, Adams E, Lavery A. Measuring Community Vulnerability to Natural and Anthropogenic Hazards: The Centers for Disease Control and Prevention's Social Vulnerability Index. J Environ Health. 2018 Jun;80(10):34-6.

13. Colen CG, Ramey DM, Cooksey EC, Williams DR. Racial disparities in health among nonpoor African Americans and Hispanics: The role of acute and chronic discrimination. Soc Sci Med. 2018 Feb;199:167-80.

14. Short SE, Mollborn S. Social Determinants and Health Behaviors: Conceptual Frames and Empirical Advances. Curr Opin Psychol. 2015 Oct;5:78-84.

15. de Wolff MG, Backhausen MG, Iversen ML, Bendix JM, Rom AL, Hegaard HK. Prevalence and predictors of maternal smoking prior to and during pregnancy in a regional Danish population: a cross-sectional study. Reproductive Health. 2019 Jun 14;16(1):82.

16. Kandel DB, Griesler PC, Schaffran C. Educational Attainment and Smoking Among Women: Risk Factors and Consequences for Offspring. Drug Alcohol Depend. 2009 Oct 1;104(Suppl 1):S24-33.

17. Gibson-Davis CM, Brooks-Gunn J. Couples' Immigration Status and Ethnicity as Determinants of Breastfeeding. Am J Public Health. 2006 Apr;96(4):641-6.

18. Social Determinants of Initiation, Duration and Exclusivity of Breastfeeding at the Population Level [Internet]. [cited 2021 Oct 4]. Available from: https://www.ncbi.nIm.nih.gov/pmc/articles/PMC6979909/

19. Jones KM, Power ML, Queenan JT, Schulkin J. Racial and Ethnic Disparities in Breastfeeding. Breastfeed Med. 2015 May 1;10(4):186-96.

20. Macharia PM, Ray N, Giorgi E, Okiro EA, Snow RW. Defining service catchment areas in low-resource settings. BMJ Global Health. 2021 Jul 1;6(7):e006381.

21. UF Health Shands Children's Hospital is No. 1 in Florida, nationally ranked in eight medical specialties [Internet]. UF Health, University of Florida Health. 2021 [cited 2021 Sep 21]. Available from: https://ufhealth.org/news/2021/ufhealth-shands-children-s-hospital-no-1-florida-nationally-ranked-eight-medical

22. Relationship Files [Internet]. [cited 2021 May 23]. Available from: https://www.census.gov/geographies/referencefiles/2010/geo/relationship-files.html\#par_textimage_674173622

23. FLHealthCHARTS.com: Home [Internet]. [cited 2021 May 23]. Available from: http://www.flhealthcharts.com/Charts/

24. R: The R Project for Statistical Computing [Internet]. [cited 2021 Sep 7]. Available from: https://www.r-project.org/

25. Remington PL, Catlin BB, Gennuso KP. The County Health Rankings: rationale and methods. Population Health Metrics. 2015 Apr 17;13(1):11.

26. Statutes \& Constitution:View Statutes: Online Sunshine [Internet]. [cited 2021 May 23]. Available from: http://www.leg.state.fl.us/Statutes/index.cfm?App_mode=Display_Statute\&Search_String=\&URL=02000299/0288/Sections/0288.0656.html

27. Wickham H, Chang W, Henry L, Pedersen TL, Takahashi K, Wilke C, et al. ggplot2: Create Elegant Data Visualisations Using the Grammar of Graphics [Internet]. 2021 [cited 2021 Oct 16]. Available from: https://CRAN.Rproject.org/package=ggplot2

28. grid package - RDocumentation [Internet]. [cited 2021 Jun 7]. Available from: https://www.rdocumentation.org/packages/grid/versions/3.6.2

29. Johnson ME, Brems C, Warner TD, Roberts LW. Rural-urban health care provider disparities in Alaska and New Mexico. Adm Policy Ment Health. 2006 Jul;33(4):504-7. 
30. Joynt KE, Harris Y, Orav EJ, Jha AK. Quality of care and patient outcomes in critical access rural hospitals. JAMA. 2011 Jul 6;306(1):45-52.

31. Ingram DD, Franco SJ. 2013 NCHS Urban-Rural Classification Scheme for Counties. Vital Health Stat 2. 2014 Apr; (166):1-73.

32. Lisonkova S, Haslam MD, Dahlgren L, Chen I, Synnes AR, Lim KI. Maternal morbidity and perinatal outcomes among women in rural versus urban areas. CMAJ. 2016 Dec 6;188(17-18):E456-65.

33. Anderson TJ, Saman DM, Lipsky MS, Lutfiyya MN. A cross-sectional study on health differences between rural and non-rural U.S. counties using the County Health Rankings. BMC Health Serv Res. 2015 Oct 1;15(1):441.

34. Maternity Care Deserts Report [Internet]. [cited 2021 May 23]. Available from: https://www.marchofdimes.org/research/maternity-care-deserts-report.aspx

35. Matthews TJ, MacDorman MF. Infant mortality statistics from the 2010 period linked birth/infant death data set. Natl Vital Stat Rep. 2013 Dec 18;62(8):1-26.

36. Neutens T. Accessibility, equity and health care: review and research directions for transport geographers. Journal of Transport Geography. 2015;43(C):14-27.

37. Improving Access to Maternal Health Care in Rural Communities [Internet]. Maternal Health Learning and Innovation Center. [cited 2021 Aug 19]. Available from: https://maternalhealthlearning.org/resources/improving-access-tomaternal-health-care-in-rural-communities/

38. Hierarchical two-step floating catchment area (2SFCA) method: measuring the spatial accessibility to hierarchical healthcare facilities in Shenzhen, China | International Journal for Equity in Health | Full Text [Internet]. [cited 2021 Aug 23]. Available from: https://equityhealthj.biomedcentral.com/articles/10.1186/s12939-020-01280-7

39. Gandy RJ. The Calculation of Catchment Populations within the National Health Service. Journal of the Royal Statistical Society Series D (The Statistician). 1979;28(1):29-37.

40. Graham GN. Why Your ZIP Code Matters More Than Your Genetic Code: Promoting Healthy Outcomes from Mother to Child. Breastfeeding Medicine. 2016 Aug 11;11(8):396-7.

41. Rural-Urban Differences In Severe Maternal Morbidity And Mortality In The US, 2007-15 | Health Affairs [Internet]. [cited 2021 Dec 2]. Available from: https://www.healthaffairs.org/doi/10.1377/hlthaff.2019.00805

42. Berg CJ, Harper MA, Atkinson SM, Bell EA, Brown HL, Hage ML, et al. Preventability of pregnancy-related deaths: results of a state-wide review. Obstet Gynecol. 2005 Dec;106(6):1228-34.

43. Geller SE, Koch AR, Martin NJ, Rosenberg D, Bigger HR, Illinois Department of Public Health Maternal Mortality Review Committee Working Group. Assessing preventability of maternal mortality in Illinois: 2002-2012. Am J Obstet Gynecol. 2014 Dec;211(6):698.e1-11.

44. Petersen EE. <em>Vital Signs</em>: Pregnancy-Related Deaths, United States, 2011-2015, and Strategies for Prevention, 13 States, 2013-2017. MMWR Morb Mortal Wkly Rep [Internet]. 2019 [cited 2021 Dec 2];68. Available from: https://www.cdc.gov/mmwr/volumes/68/wr/mm6818e1.htm

45. BERNET P, GUMUS G, VISHWASRAO S. Maternal Mortality and Public Health Programs: Evidence from Florida. Milbank Q. 2020 Mar;98(1):150-71.

46. Beyond The Preventing Maternal Deaths Act: Implementation And Further Policy Change | Health Affairs Blog [Internet]. [cited 2021 Dec 2]. Available from: https://www.healthaffairs.org/do/10.1377/hblog20190130.914004/full/

47. Bjørnholt SM, Leite M, Albieri V, Kjaer SK, Jensen A. Maternal smoking during pregnancy and risk of stillbirth: results from a nationwide Danish register-based cohort study. Acta Obstetricia et Gynecologica Scandinavica.

2016;95(11):1305-12.

48. Stock SJ, Bauld L. Maternal smoking and preterm birth: An unresolved health challenge. PLOS Medicine. 2020 Sep 14;17(9):e1003386.

Page 13/18 
49. Salihu HM, Wilson RE. Epidemiology of prenatal smoking and perinatal outcomes. Early Human Development. 2007 Nov 1;83(11):713-20.

50. Strully KW, Rehkopf DH, Xuan Z. Effects of Prenatal Poverty on Infant Health: State Earned Income Tax Credits and Birth Weight. Am Sociol Rev. 2010 Aug 11;75(4):534-62.

51. Greer FR. Long-term Adverse Outcomes of Low Birth Weight, Increased Somatic Growth Rates, and Alterations of Body Composition in the Premature Infant: Review of the Evidence. Journal of Pediatric Gastroenterology and Nutrition. 2007 Dec;45:S147.

52. Young RL, Weinberg J, Vieira V, Aschengrau A, Webster TF. A multilevel non-hierarchical study of birth weight and socioeconomic status. International Journal of Health Geographics. 2010 Jul 9;9(1):36.

53. Cohen SS, Alexander DD, Krebs NF, Young BE, Cabana MD, Erdmann P, et al. Factors Associated with Breastfeeding Initiation and Continuation: A Meta-Analysis. J Pediatr. 2018 Dec;203:190-196.e21.

54. Georgia-Uses-Telehealth-to-Improve-Maternal-and-Child-Health-Outcomes.pdf [Internet]. [cited 2021 Jun 19]. Available from: https://astho.org/Maternal-and-Child-Health/Georgia-Uses-Telehealth-to-Improve-Maternal-and-Child-HealthOutcomes/

55. Overview [Internet]. UF Health, University of Florida Health. 2020 [cited 2021 May 23]. Available from: https://ufhealth.org/uf-health-telehealth/overview

56. DeNicola N, Grossman D, Marko K, Sonalkar S, Butler Tobah YS, Ganju N, et al. Telehealth Interventions to Improve Obstetric and Gynecologic Health Outcomes: A Systematic Review. Obstet Gynecol. 2020 Feb;135(2):371-82.

57. Leyk S, Balk D, Jones B, Montgomery MR, Engin $H$. The heterogeneity and change in the urban structure of metropolitan areas in the United States, 1990-2010. Sci Data. 2019 Dec 16;6(1):321.

\section{Figures}
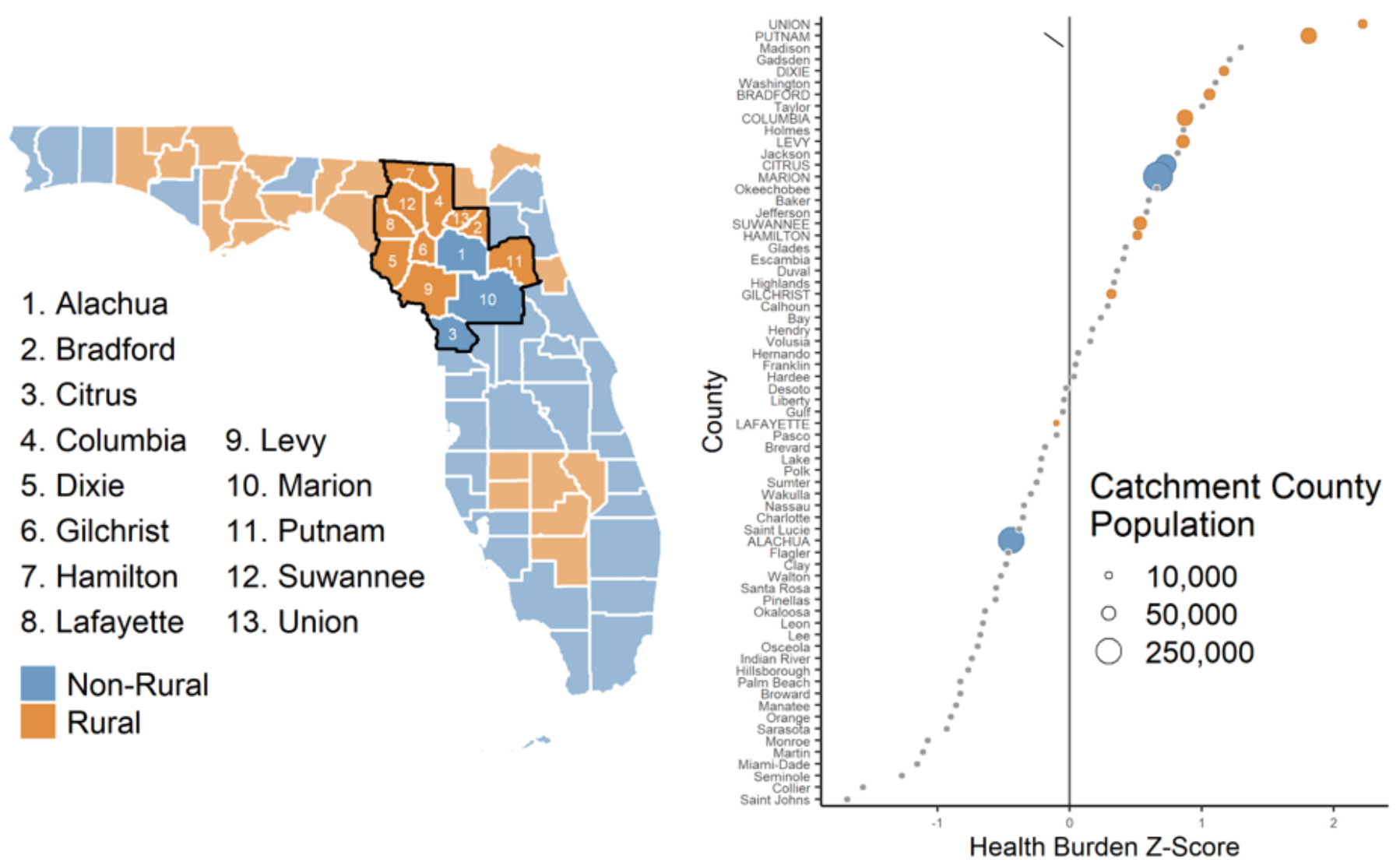


\section{Figure 1}

\section{Rurality by Florida County and Associated County-Health Rankings Z-Scores.}

Map of the rurality of 67 Florida counties according to Florida Statues rurality designations with UFHPCA boundary outlined in black (left) and County Health Rankings Health Outcomes z-scores (right), where positive z-scores represent a greater health burden compared to the average of counties in Florida. Counties outside the UFHPCA are depicted as grey circles in the right. Only two of the thirteen catchment counties (Alachua and Lafayette) had higher health outcomes scores than the average of Florida counties, indicating that the UFHPCA as a whole has a higher health burden than the state. Three catchment counties (Union, Putnam, and Dixie) were in the top five highest health burden counties in Florida.
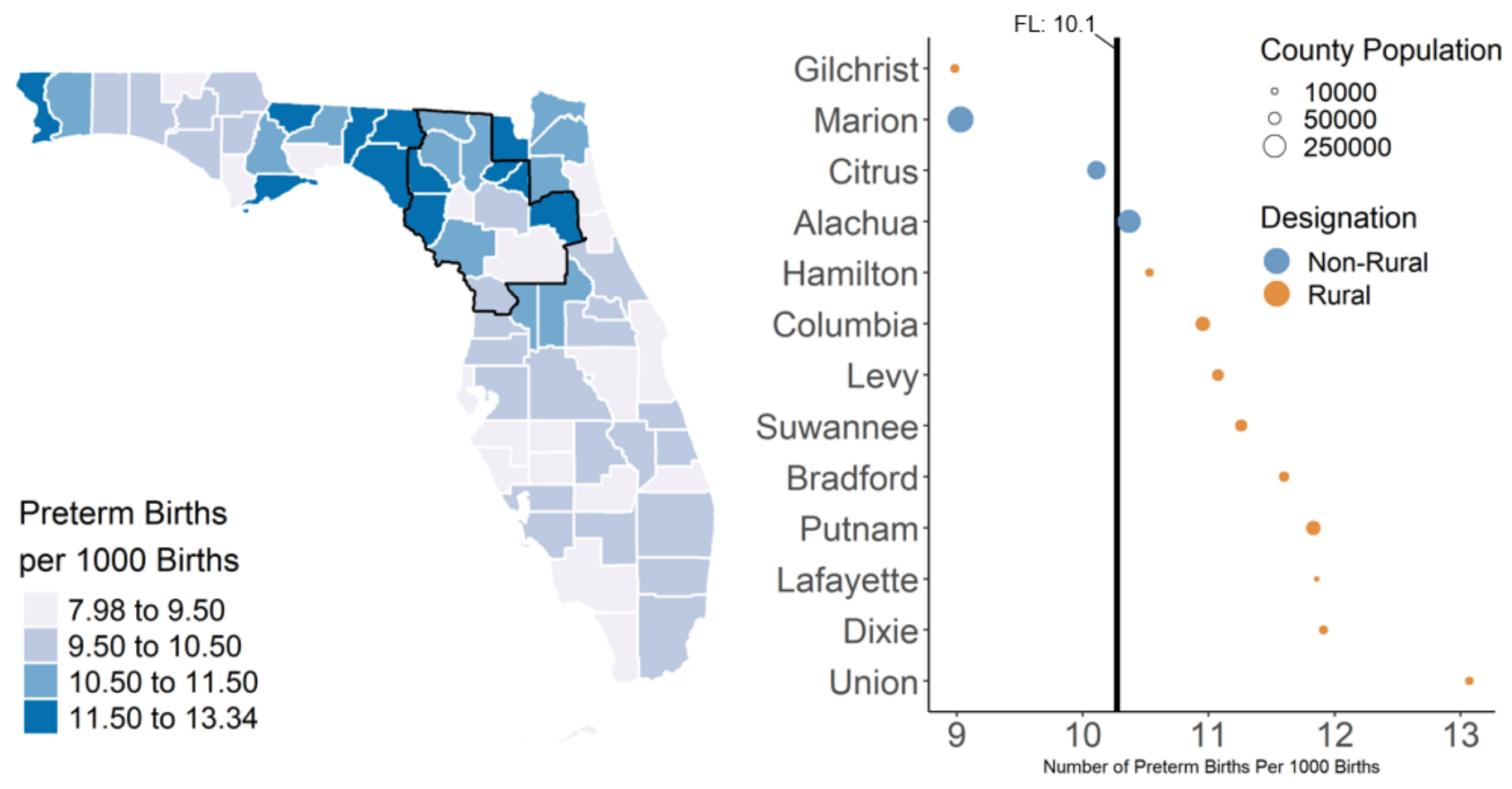

Figure 2

\section{Rates of Preterm Births in Florida and the UFHPCA}

Statewide map of preterm births with UF Health Perinatal Catchment Area (left; outlined in black). The thirteen catchment counties are ordered by rate where the statewide rate (10.1\% from June 2011 - April 2017) is denoted by the vertical black line (right). Rates are weighted by county-level annual population. Within the UFHPCA ten out of thirteen counties had preterm birth rates above the statewide rate, indicating higher levels of health burden within the catchment. 

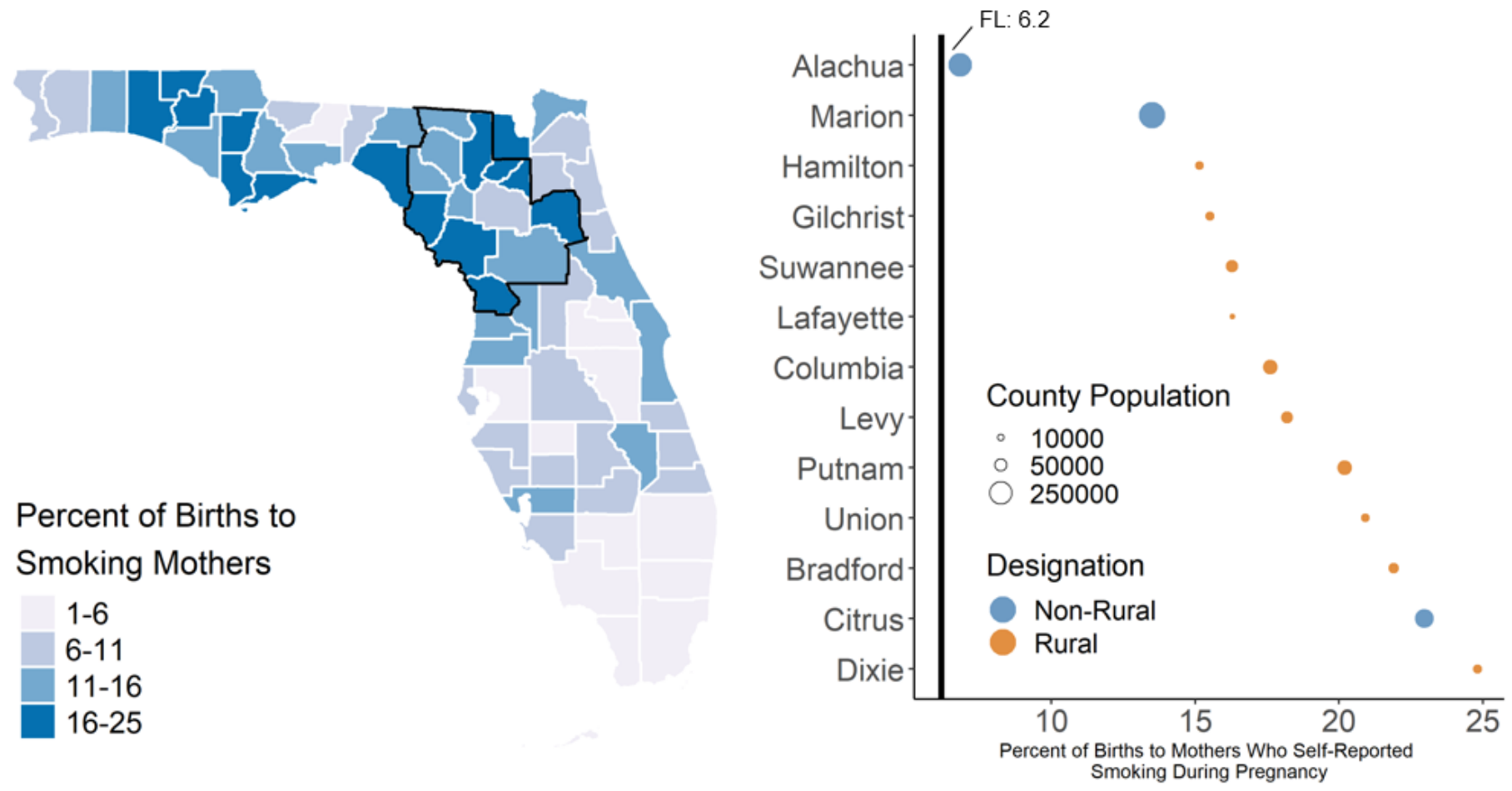

Figure 3

Rates of Maternal Smoking during Pregnancy in Florida and the UFHPCA. Statewide map of the percent of births to mothers smoked during pregnancy with the UFHPCA (left; outlined in black) and the thirteen catchment counties ordered by smoking rate where the statewide rate (6.2\% between June 2011 - April 2017) is denoted by the vertical black line (right). Rates are weighted by county-level annual population. All thirteen catchment counties had maternal smoking rates during pregnancy above the statewide rate.
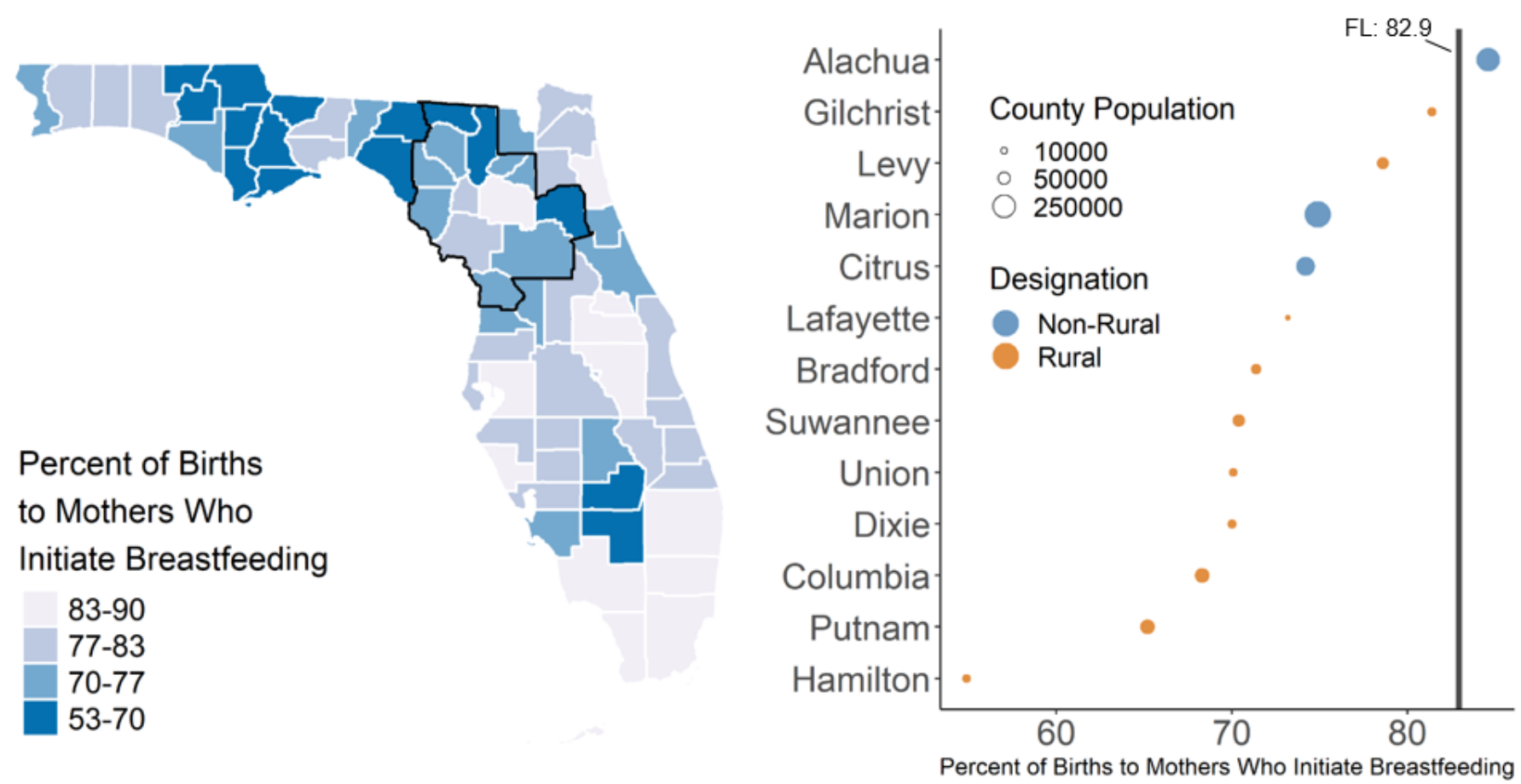


\section{Figure 4}

\section{Rates of Breastfeeding Initiation in Florida and the UFHPCA}

Statewide map of county level breastfeeding initiation rates with the UFHPCA (left; outlined in black) and the thirteen catchment counties ordered by breastfeeding initiation rate where the statewide rate (82.9\% between June 2011 - April 2017 ) is denoted by the vertical black line (right). Rates are weighted by county-level annual population. Within the catchment, all counties except Alachua County had rates below the statewide rate, indicating higher levels of health burden within the catchment.
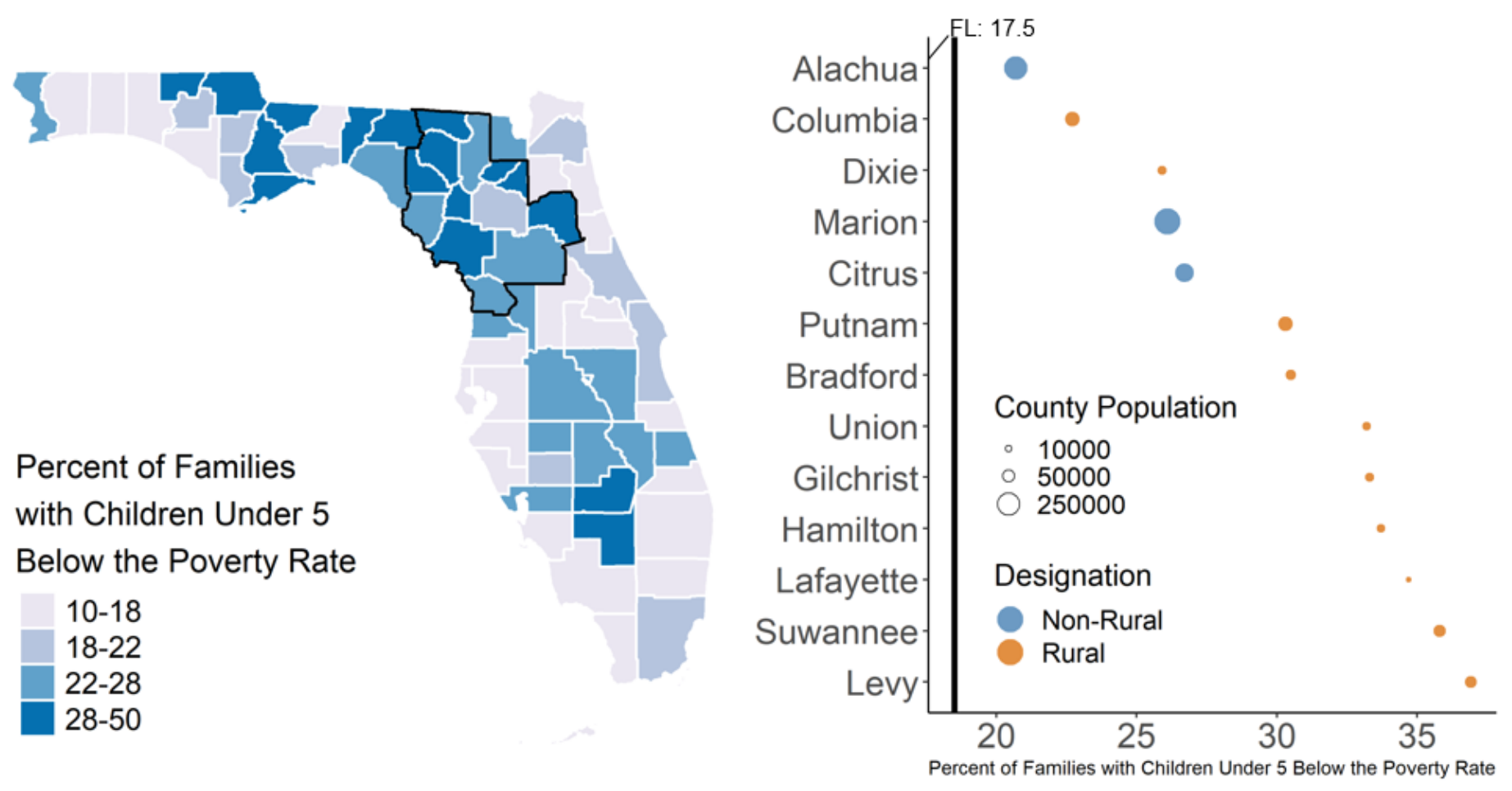

\section{Figure 5}

\section{Poverty Rate for Families with Children Under 5 in Florida and the UFHPCA}

Statewide map of families with related children under the age of 5 living below the poverty rate with the UFHPCA (left; outlined in black). The thirteen catchment counties are ordered by rate where the statewide rate (17.5\% from June 2011 April 2017) is denoted by the vertical black line (right). Rates are weighted by county-level annual population. Within the UFHPCA all counties had poverty rates above the statewide rate, indicating higher levels of health burden within the catchment. 

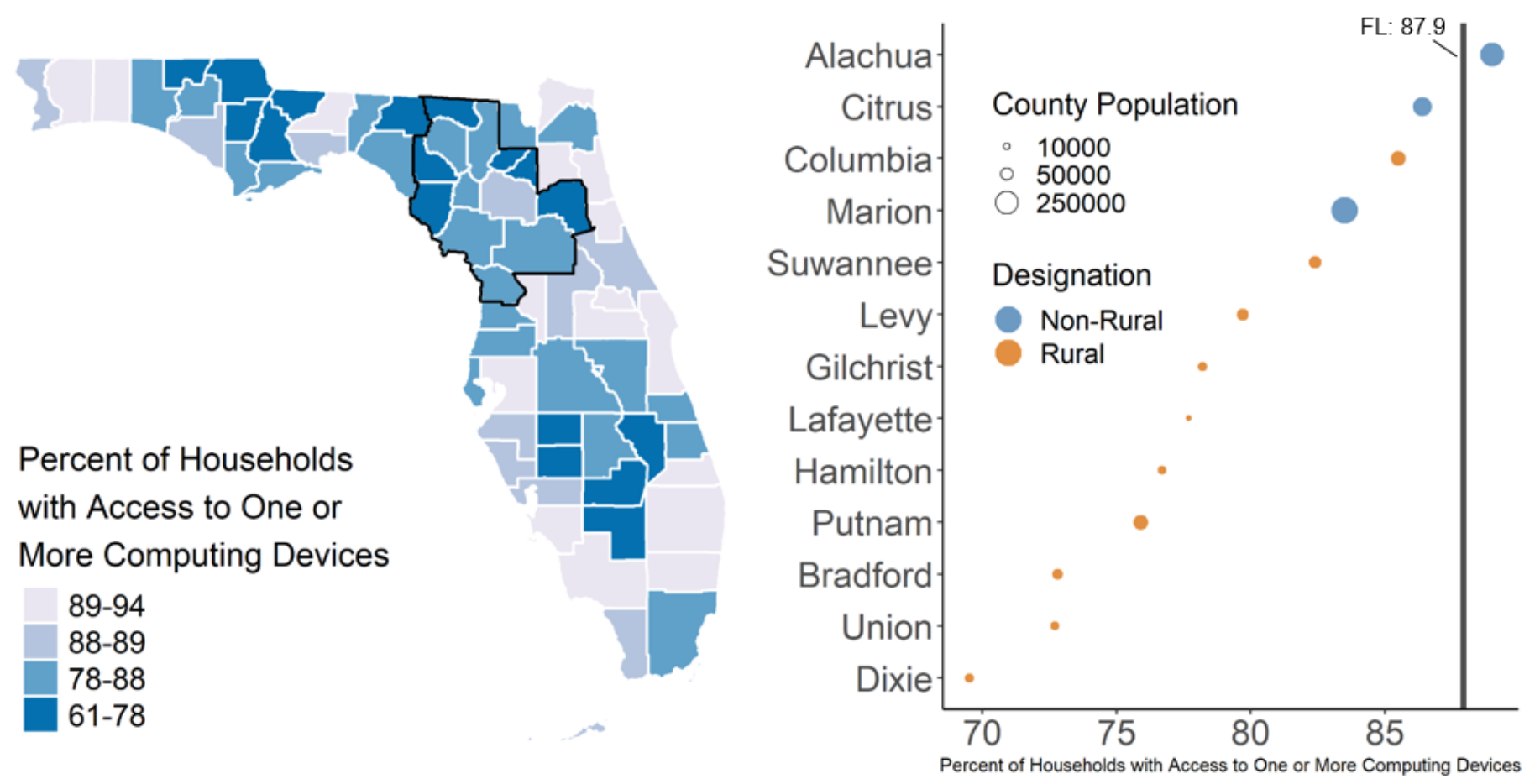

Figure 6

\section{Rates of Access to Household Computing Devices in Florida and the UFHPCA in 2017}

Statewide map of rates of computing device access within households in the UFHPCA (left; outlined in black). The thirteen catchment counties are ordered by household rates of access to one or more computing device, where the 2017 statewide rate $(87.9 \%)$ is denoted by the vertical black line (right). Within the UFHPCA all counties except Alachua County had computing device access rates below the statewide rate, indicating higher levels of health burden within the catchment.

\section{Supplementary Files}

This is a list of supplementary files associated with this preprint. Click to download.

- sf1.png 